\title{
REMARKS ON THE $(C,-1)$-SUMMABILITY OF THE DISTRIBUTION OF ZEROS OF ORTHOGONAL POLYNOMIALS
}

\author{
PAUL NEVAI AND WALTER VAN ASSCHE
}

(Communicated by J. Marshall Ash)

\begin{abstract}
Given $x_{1}<x_{2}<\cdots<x_{n}$ and $y_{1}<y_{2}<\cdots<y_{n-1}$, two interlacing sequences of real numbers, the rectangular diagram for these numbers is a continuous piecewise linear function with slopes \pm 1 and with $n$ local minima at the points $x_{i}$ and $n-1$ local maxima at the points $y_{j}$. Recently, S. Kerov determined the asymptotic behavior of the rectangular diagrams associated with the zeros of two consecutive orthogonal polynomials for which the coefficients in the three-term recurrence relation converge. The purpose of this note is to show how this result of S. Kerov and even some of its generalizations follow directly from certain $(C,-1)$-summability results on distribution of zeros of orthogonal polynomials proved by us some time ago.
\end{abstract}

\section{INTRODUCTION}

Let $\alpha$ be a non-negative finite Borel measure with finite moments on the real line, and let $P_{n}(\alpha)$ be the corresponding monic orthogonal polynomials with zeros $x_{1, n}(\alpha)<x_{2, n}(\alpha)<\cdots<x_{n, n}(\alpha)$. Consider the distribution function

$$
F_{n}(\alpha, x)=\sum_{j=1}^{n} U\left(x-x_{j, n}(\alpha)\right),
$$

where $U$ is the Heaviside function defined by

$$
U(x)= \begin{cases}0, & \text { if } x<0 \\ 1, & \text { if } x \geq 0\end{cases}
$$

Then $F_{n}(\alpha)$ is a nondecreasing piecewise constant function with jumps of size 1 at each zero $x_{j, n}(\alpha)$.

Definition. Given $0 \leq a<\infty$ and $b \in \mathbb{R}$, we say that the orthogonality measure $\alpha \in \mathbf{M}(b, a)$ if $\lim _{n \rightarrow \infty} a_{n}(\alpha)=a / 2$ and $\lim _{n \rightarrow \infty} b_{n}(\alpha)=b$ where $a_{n}(\alpha)>0$ and $b_{n}(\alpha) \in \mathbb{R}$ are the recursion coefficients in three-term recurrence

$$
\left(x-b_{n}(\alpha)\right) P_{n}(\alpha, x)=P_{n+1}(\alpha, x)+a_{n}^{2}(\alpha) P_{n-1}(\alpha, x) .
$$

Received by the editors September 11, 1992 and, in revised form, February 11, 1993.

1991 Mathematics Subject Classification. Primary 42C05.

Key words and phrases. Orthogonal polynomials, zeros, rectangular diagrams.

This material is based upon work supported by the National Science Foundation under Grant No. DMS-9024901 (P. N.), by NATO under Grant No. CRG.870806 (P. N.), and by a research grant of the Belgian National Science Foundation (W. V. A.). The second author is a research associate of the Belgian National Fund for Scientific Research. 
Define the function $\omega_{n}(\alpha): \mathbb{R} \mapsto \mathbb{R}$ by

$$
\begin{aligned}
\omega_{n}(\alpha, t) \stackrel{\text { def }}{=} & \int_{x_{1, n}(\alpha)}^{t}\left[2 F_{n}(\alpha, x)-2 F_{n-1}(\alpha, x)-1\right] d x \\
& -\int_{x_{1, n}(\alpha)}^{x_{n, n}(\alpha)}\left[F_{n}(\alpha, x)-F_{n-1}(\alpha, x)-1\right] d x
\end{aligned}
$$

Then, obviously,

$$
\omega_{n}^{\prime}(\alpha, t)=2 F_{n}(\alpha, t)-2 F_{n-1}(\alpha, t)-1, \quad t \notin\left\{x_{j, n}(\alpha)\right\} \cup\left\{x_{j, n-1}(\alpha)\right\}
$$

In particular, taking into account the interlacing property $x_{j, n}(\alpha)<x_{j, n-1}(\alpha)<$ $x_{j+1, n}(\alpha)$,

$$
\omega_{n}^{\prime}(\alpha, t)= \begin{cases}-1, & t<x_{1, n}(\alpha), \\ 2 j-2(j-1)-1=1, & x_{j, n}(\alpha)<t<x_{j, n-1}(\alpha), \\ 2 j-2 j-1=-1, & x_{j, n-1}(\alpha)<t<x_{j+1, n}(\alpha), \\ 1, & t>x_{n, n}(\alpha),\end{cases}
$$

so that $\omega_{n}(\alpha)$ is continuous and piecewise linear with slopes \pm 1 , with minima at $x_{j, n}(\alpha)$, and maxima at $x_{j, n-1}(\alpha)$, and

$$
\begin{array}{r}
\omega_{n}(\alpha, t)=\left|t-x_{n, n}(\alpha)+\int_{x_{1, n}(\alpha)}^{x_{n, n}(\alpha)}\left[F_{n}(\alpha, x)-F_{n-1}(\alpha, x)\right] d x\right| \\
t \notin\left[x_{1, n}(\alpha), x_{n, n}(\alpha)\right],
\end{array}
$$

that is, using S. Kerov's terminology [2, (i) and (ii) on p. 1, and formula (1.6) on p. 2] (cf. [3], [4], and [5]), $\omega_{n}(\alpha)$ is precisely the rectangular diagram describing the separation of the zeros of $P_{n-1}(\alpha)$ and $P_{n}(\alpha)$.

Notice that

$$
\begin{array}{r}
\int_{x_{1, n}(\alpha)}^{x_{n, n}(\alpha)} F_{n}(\alpha, x) d x=\sum_{j=1}^{n} \int_{x_{1, n}(\alpha)}^{x_{n, n}(\alpha)} U\left(x-x_{j, n}(\alpha)\right) d x \\
=\sum_{j=1}^{n}\left(x_{n, n}(\alpha)-x_{j, n}(\alpha)\right)=n x_{n, n}(\alpha)-\sum_{j=1}^{n} x_{j, n}(\alpha),
\end{array}
$$

and, similarly,

$$
\begin{aligned}
& \int_{x_{1, n}(\alpha)}^{x_{n, n}(\alpha)} F_{n-1}(\alpha, x) d x=\sum_{j=1}^{n-1} \int_{x_{1, n}(\alpha)}^{x_{n, n}(\alpha)} U\left(x-x_{j, n-1}(\alpha)\right) d x \\
& =\sum_{j=1}^{n-1}\left(x_{n, n}(\alpha)-x_{j, n-1}(\alpha)\right)=(n-1) x_{n, n}(\alpha)-\sum_{j=1}^{n-1} x_{j, n-1}(\alpha),
\end{aligned}
$$


so that

$$
\begin{aligned}
\omega_{n}(\alpha, t)= & \int_{x_{1, n}(\alpha)}^{t}\left[2 F_{n}(\alpha, x)-2 F_{n-1}(\alpha, x)-1\right] d x \\
& -x_{1, n}(\alpha)+\sum_{j=1}^{n} x_{j, n}(\alpha)-\sum_{j=1}^{n-1} x_{j, n-1}(\alpha) \\
= & \int_{x_{1, n}(\alpha)}^{t}\left[2 F_{n}(\alpha, x)-2 F_{n-1}(\alpha, x)-1\right] d x \\
& -x_{1, n}(\alpha)+b_{n-1}(\alpha), \quad t \in \mathbb{R},
\end{aligned}
$$

and

$$
\omega_{n}(\alpha, t)=\left|t+\sum_{j=1}^{n-1} x_{j, n-1}(\alpha)-\sum_{j=1}^{n} x_{j, n}(\alpha)\right|=\left|t-b_{n-1}(\alpha)\right|
$$

$$
t \notin\left[x_{1, n}(\alpha), x_{n, n}(\alpha)\right]
$$

where we used the formula

$$
\sum_{j=1}^{n} x_{j, n}(\alpha)=\sum_{j=0}^{n-1} b_{j}(\alpha)
$$

which follows directly from the Jacobi-matrix interpretation of orthogonal polynomials.

S. Kerov [2, Theorem 1, p. 2] (cf. [3, Theorem 1] and [4, Theorem 1.5]) proved the following

Theorem 1. Suppose $\alpha \in \mathbf{M}(b, a)$ with $a>0$. Then

$$
\lim _{n \rightarrow \infty} \omega_{n}(\alpha, t)=\left\{\begin{array}{cc}
\frac{2 a}{\pi}\left(\frac{t-b}{a} \arcsin \left(\frac{t-b}{a}\right)+\sqrt{1-\left(\frac{t-b}{a}\right)^{2}}\right), \\
t \in[b-a, b+a], \\
|t-b|, \quad t \notin[b-a, b+a],
\end{array}\right.
$$

uniformly for $t \in \mathbb{R}$.

The purpose of this note is to show how Theorem 1 and even some of its generalizations follow directly from certain $(C,-1)$-summability results on the distribution of zeros of orthogonal polynomials proved by us some time ago (see, e.g., $[6,10]$ ).

\section{A genERALIZATION OF KeROV'S THEOREM}

First, we point out that [6, Theorem 10 , p. 350] can be generalized from continuously differentiable functions to absolutely continuous ones (and from $\mathrm{M}(b, a)$ with $a>0$ to $\mathrm{M}(b, a)$ with $a \geq 0)$ as follows.

Theorem 2. Suppose $\alpha \in \mathbf{M}(b, a)$ with $a \geq 0$. If $f$ is absolutely continuous in an interval, say, $\Delta$ such that $\operatorname{supp}(\alpha) \subseteq \Delta$, then

(6) $\lim _{n \rightarrow \infty} \sum_{j=1}^{n} f\left(x_{j, n}(\alpha)\right)-\sum_{j=1}^{n-1} f\left(x_{j, n-1}(\alpha)\right)= \begin{cases}\frac{1}{\pi} \int_{b-a}^{b+a} \frac{f(t) d t}{\sqrt{a^{2}-(t-b)^{2}}}, & \text { if } a>0, \\ f(b), & \text { if } a=0 .\end{cases}$ 
For $a>0$, this is a straightforward consequence of [6, Theorem 10, p. 350]. Nevertheless, the simplicity of the following proof deserves special attention. For the proof of Theorem 2, we need the following

Lemma 3. Let $\rho$ be given by $2 \rho(z)=z+\sqrt{z^{2}-1}$ where we choose that branch of the square root which is positive for $z>1$. If $\alpha \in \mathbf{M}(b, a)$ with $a \geq 0$, then the corresponding monic orthogonal polynomials $P_{n}(\alpha)$ satisfy

$$
\lim _{n \rightarrow \infty} \frac{P_{n}(\alpha, z)}{P_{n-1}(\alpha, z)}= \begin{cases}a \rho\left(\frac{z-b}{a}\right), & \text { if } a>0, \\ z-b, & \text { if } a=0,\end{cases}
$$

uniformly on every compact set outside the support of $\alpha$.

Proof of Lemma 3. For $a>0$ this Poincare type lemma has been proved in [7, Theorem 4.1.13, p. 33], whereas for $a=0$ we argue as follows. Consider the functionals $G_{n}(\alpha)$ given by

$$
G_{n}(\alpha, g)=\sum_{k=1}^{n} \lambda_{k, n}(\alpha) g\left(x_{k, n}(\alpha)\right) p_{n-1}^{2}\left(\alpha, x_{k, n}(\alpha)\right)
$$

where $p_{n}(\alpha)$ denotes the orthonormal polynomial associated with $\alpha$ and $\lambda_{k, n}(\alpha)$ are the Cotes numbers of the corresponding Gauss-Jacobi quadrature formula (cf. [8, formula (3.4.1), p. 47]). For $i=0,1$, and 2 , let $g_{i}$ be given by $g_{i}(x) \equiv x^{i}$. Then, by the recurrence formula (1) and the Gauss-Jacobi quadrature formula, we have $G_{n}\left(\alpha, g_{0}\right) \equiv 1, G_{n}\left(\alpha, g_{1}\right) \equiv b_{n-1}(\alpha)$, and $G_{n}\left(\alpha, g_{2}\right) \equiv$ $b_{n-1}^{2}(\alpha)+a_{n-1}^{2}(\alpha)$, so that $\lim _{n \rightarrow \infty} G_{n}\left(\alpha, g_{i}\right)=g_{i}(b)$ for $i=0,1$, and 2 . Hence, by the Bohman-Korovkin theorem on the convergence of monotone (or positive) operators (cf. [1, p. 67]), we have $\lim _{n \rightarrow \infty} G_{n}(\alpha, g)=g(b)$ for every continuous function $g$. In particular, if $g_{z}$ is given by $g_{z}(x)=(z-x)^{-1}$, then, by the Stieltjes-Vitali theorem, $\lim _{n \rightarrow \infty} G_{n}\left(\alpha, g_{z}\right)=g_{z}(b)$ locally uniformly outside the derived set of the set of all zeros of all $p_{n}(\alpha)$ 's. According to [7, Theorem 3.3.8, p. 24], for $\alpha \in \mathbf{M}(b, a)$, the derived set of the set of all zeros of all $p_{n}(\alpha)$ 's is precisely the support of $\alpha .^{1}$ Finally, we observe that $G_{n}\left(\alpha, g_{z}\right) \equiv \frac{P_{n-1}(\alpha, z)}{P_{n}(\alpha, z)}$ (cf. [8, formulas (3.3.9), (3.3.10), and (3.4.7), pp. 4748]). Hence, (7) follows for $a=0$ as well.

Proof of Theorem 2. We have

$$
\sum_{j=1}^{n} f\left(x_{j, n}(\alpha)\right)-\sum_{j=1}^{n-1} f\left(x_{j, n-1}(\alpha)\right)=\sum_{j=2}^{n} \int_{x_{j-1, n-1}(\alpha)}^{x_{j, n}(\alpha)} f^{\prime}(t) d t+f\left(x_{1, n}(\alpha)\right),
$$

so that

$$
\left|\sum_{j=1}^{n} f\left(x_{j, n}(\alpha)\right)-\sum_{j=1}^{n-1} f\left(x_{j, n-1}(\alpha)\right)\right| \leq \int_{\Delta}\left|f^{\prime}(t)\right| d t+\sup _{t \in \Delta}|f(t)|,
$$

since the zeros $\left\{x_{j, n-1}(\alpha)\right\}_{j=1}^{n-1}$ and $\left\{x_{j, n}(\alpha)\right\}_{j=1}^{n}$ interlace and belong to $\Delta$. Hence, we need to prove (6) for sufficiently smooth, say, entire functions only.

\footnotetext{
${ }^{1}$ For measures not belonging to $\mathrm{M}(b, a)$ this no longer holds; a simple counterexample being the Lebesgue measure supported in the intervals $[-2,-1]$ and $[1,2]$.
} 
However, if $f$ is entire, then we can write

$$
\begin{aligned}
& \sum_{j=1}^{n} f\left(x_{j, n}(\alpha)\right)-\sum_{j=1}^{n-1} f\left(x_{j, n-1}(\alpha)\right) \\
& \quad=\frac{1}{2 \pi i} \int_{\Gamma} f(z)\left[\frac{P_{n}^{\prime}(\alpha, z)}{P_{n}(\alpha, z)}-\frac{P_{n-1}^{\prime}(\alpha, z)}{P_{n-1}(\alpha, z)}\right] d z \\
& \quad=\frac{1}{2 \pi i} \int_{\Gamma} f(z)\left[\log \frac{P_{n}(\alpha, z)}{P_{n-1}(\alpha, z)}\right]^{\prime} d z
\end{aligned}
$$

where $\Gamma$ is a Jordan curve containing $\Delta$ in its interior. Hence, by Lemma 3 and the Stieltjes-Vitali theorem, (6) follows for entire functions, and then, by (8), for all absolutely continuous functions as well.

A generalized Kerov function is probably best formulated in terms of a natural generalization of $\omega_{n}(\alpha, t)$ in (2) given by

$$
\Omega_{n}(\alpha, g) \stackrel{\text { def }}{=} \int_{x_{1, n}(\alpha)}^{x_{n, n}(\alpha)}\left[2 F_{n}(\alpha, x)-2 F_{n-1}(\alpha, x)-1\right] g(x) d x .
$$

Then, using (4), for $t \in\left[x_{1, n}(\alpha), x_{n, n}(\alpha)\right]$, Kerov's rectangular diagram $\omega_{n}(\alpha)$ can be written as

$$
\omega_{n}(\alpha, t)=\Omega_{n}\left(\alpha, g_{t}\right)-x_{1, n}(\alpha)+b_{n-1}(\alpha)
$$

where $g_{t}$ is the characteristic function of $(-\infty, t)$, and Theorem 1 is a partial case of the following

Theorem 4. Suppose $\alpha \in \mathbf{M}(b, a)$ with $a \geq 0$. If $g$ is integrable in an interval, say, $\Delta$ such that $\operatorname{supp}(\alpha) \subseteq \Delta$, then

$$
\lim _{n \rightarrow \infty} \Omega_{n}(\alpha, g)=\left\{\begin{array}{cc}
\frac{2}{\pi} \int_{b-a}^{b+a} g(t) \arcsin \left(\frac{t-b}{a}\right) d t-\int_{\xi_{L}(\alpha)}^{b-a} g(t) d t+\int_{b+a}^{\xi_{R}(\alpha)} g(t) d t, \\
\text { if } a>0, \\
-\int_{\xi_{L}(\alpha)}^{b} g(t) d t+\int_{b}^{\xi_{R}(\alpha)} g(t) d t, & \text { if } a=0,
\end{array}\right.
$$

where $\xi_{L}(\alpha) \stackrel{\text { def }}{=} \lim _{n \rightarrow \infty} x_{1, n}(\alpha)$ and $\xi_{R}(\alpha) \stackrel{\text { def }}{=} \lim _{n \rightarrow \infty} x_{n, n}(\alpha)$.

Proof of Theorem 2. Let $G$ be an antiderivative of $g$. Then

$$
\begin{aligned}
\int_{x_{1, n}(\alpha)}^{x_{n, n}(\alpha)} F_{n}(\alpha, x) g(x) d x & =\sum_{j=1}^{n} \int_{x_{1, n}(\alpha)}^{x_{n, n}(\alpha)} U\left(x-x_{j, n}(\alpha)\right) g(x) d x \\
& =\sum_{j=1}^{n}\left[G\left(x_{n, n}(\alpha)\right)-G\left(x_{j, n}(\alpha)\right)\right] \\
& =n G\left(x_{n, n}(\alpha)\right)-\sum_{j=1}^{n} G\left(x_{j, n}(\alpha)\right),
\end{aligned}
$$


and, similarly,

$$
\begin{aligned}
\int_{x_{1, n}(\alpha)}^{x_{n, n}(\alpha)} F_{n-1}(\alpha, x) g(x) d x & =\sum_{j=1}^{n-1} \int_{x_{1, n}(\alpha)}^{x_{n, n}(\alpha)} U\left(x-x_{j, n-1}(\alpha)\right) g(x) d x \\
& =\sum_{j=1}^{n-1}\left[G\left(x_{n, n}(\alpha)\right)-G\left(x_{j, n-1}(\alpha)\right)\right] \\
& =(n-1) G\left(x_{n, n}(\alpha)\right)-\sum_{j=1}^{n-1} G\left(x_{j, n-1}(\alpha)\right),
\end{aligned}
$$

so that

$\Omega_{n}(\alpha, g)=G\left(x_{1, n}(\alpha)\right)+G\left(x_{n, n}(\alpha)\right)-2\left(\sum_{j=1}^{n} G\left(x_{j, n}(\alpha)\right)-\sum_{j=1}^{n-1} G\left(x_{j, n-1}(\alpha)\right)\right)$.

Hence, if $a>0$, then by Theorem 2 ,

$$
\begin{aligned}
\lim _{n \rightarrow \infty} \Omega_{n}(\alpha, g)= & G\left(\xi_{L}(\alpha)\right)+G\left(\xi_{R}(\alpha)\right)-\frac{2}{\pi} \int_{b-a}^{b+a} \frac{G(t) d t}{\sqrt{a^{2}-(t-b)^{2}}} \\
= & \frac{2}{\pi} \int_{b-a}^{b+a} g(t) \arcsin \left(\frac{t-b}{a}\right) d t \\
& +G\left(\xi_{L}(\alpha)\right)-G(b-a)+G\left(\xi_{R}(\alpha)\right)-G(b+a),
\end{aligned}
$$

whereas, for $a=0$, Theorem 2 gives

$$
\begin{aligned}
\lim _{n \rightarrow \infty} \Omega_{n}(\alpha, g) & =G\left(\xi_{L}(\alpha)\right)+G\left(\xi_{R}(\alpha)\right)-2 G(b) \\
& =G\left(\xi_{L}(\alpha)\right)-G(b)+G\left(\xi_{R}(\alpha)\right)-G(b)
\end{aligned}
$$

which is equivalent to (12).

\section{UNBOUNDED RECURRENCE COEFFICIENTS}

Definition. The function $\varphi: \mathbb{R}^{+} \mapsto \mathbb{R}^{+}$is called a log-slowly varying function, if it is an increasing function for which

$$
\lim _{x \rightarrow+\infty} \varphi(x)=\infty \quad \text { and } \quad \lim _{x \rightarrow+\infty} \frac{\varphi(x+y)}{\varphi(x)}=1, \quad \forall y \in \mathbb{R},
$$

hold.

When the recurrence coefficients are unbounded, that is, when

$$
\lim _{n \rightarrow \infty}\left(a_{n}(\alpha)+\left|b_{n}(\alpha)\right|\right)=\infty
$$

then an interesting class of recurrence coefficients is the class for which the asymptotic behavior $\lim _{n \rightarrow \infty} a_{n}(\alpha) / \varphi(n)=a / 2<\infty$ and $\lim _{n \rightarrow \infty} b_{n}(\alpha) / \varphi(n)=$ $b \in \mathbb{R}$ hold, where $\varphi$ is a log-slowly varying function. For such recurrence coefficients we have the following analog of Lemma 3. 
Lemma 5. Suppose $\lim _{n \rightarrow \infty} a_{n}(\alpha) / \varphi(n)=a / 2 \geq 0$ and $\lim _{n \rightarrow \infty} b_{n}(\alpha) / \varphi(n)=$ $b \in \mathbb{R}$, where $\varphi$ is a log-slowly varying function. If $[A, B]$ is the convex hull of $[b-a, b+a] \cup\{0\}$ then the corresponding monic orthogonal polynomials satisfy

$$
\lim _{n \rightarrow \infty} \frac{1}{\varphi(n)} \frac{P_{n}(\alpha, \varphi(n) z)}{P_{n-1}(\alpha, \varphi(n) z)}= \begin{cases}a \rho\left(\frac{z-b}{a}\right), & \text { if } a>0, \\ z-b, & \text { if } a=0,\end{cases}
$$

uniformly on every compact set outside $[A, B]$. In addition, the smallest and largest zeros satisfy

$$
\lim _{n \rightarrow \infty} \frac{x_{1, n}(\alpha)}{\varphi(n)}=A \quad \text { and } \quad \lim _{n \rightarrow \infty} \frac{x_{n, n}(\alpha)}{\varphi(n)}=B,
$$

respectively.

Proof of Lemma 5. The ratio asymptotic behavior in (13) is given in [10, Theorem 2.1, p. 8]. To prove $(14)$, first we observe that

$$
\min (0, b-a) \leq \liminf _{n \rightarrow \infty} \frac{x_{1, n}(\alpha)}{\varphi(n)} \leq \limsup _{n \rightarrow \infty} \frac{x_{n, n}(\alpha)}{\varphi(n)} \leq \max (0, b+a)
$$

which follows immediately from the Gershgorin bounds

$$
\min _{0 \leq i \leq n-1}\left(b_{i}(\alpha)-a_{i}(\alpha)-a_{i+1}(\alpha)\right) \leq x_{j, n}(\alpha) \leq \max _{0 \leq i \leq n-1}\left(b_{i}(\alpha)+a_{i}(\alpha)+a_{i+1}(\alpha)\right),
$$

where $a_{0}=0$ (cf. $[11$, p. 437 and p. 455]). The ratio asymptotic behavior (13) is equivalent to

$$
\begin{aligned}
\lim _{n \rightarrow \infty} & \sum_{j=1}^{n} \lambda_{j, n}(\alpha) p_{n-1}^{2}\left(\alpha, x_{j, n}(\alpha)\right) f\left(x_{j, n}(\alpha) / \varphi(n)\right) \\
& = \begin{cases}\frac{2}{\pi a^{2}} \int_{b-a}^{b+a} f(t) \sqrt{a^{2}-(t-b)^{2}} d t, & \text { if } a>0, \\
f(b), & \text { if } a=0,\end{cases}
\end{aligned}
$$

for every continuous function $f$ in $\Delta$, where $\lambda_{j, n}(\alpha)$ are the Christoffel numbers and $p_{n}(\alpha)$ are the orthonormal polynomials (see [10, p. 9]). This means that every point in the interval $[b-a, b+a]$ is a limit point of the set of contracted zeros $\left\{x_{j, n}(\alpha) / \varphi(n)\right\}$. In particular, this implies

$$
\liminf _{n \rightarrow \infty} \frac{x_{1, n}(\alpha)}{\varphi(n)} \leq b-a \quad \text { and } \quad \limsup _{n \rightarrow \infty} \frac{x_{n, n}(\alpha)}{\varphi(n)} \geq b+a .
$$

If $0 \in[b-a, b+a]$, then $A=b-a$ and $B=b+a$ and the result in (14) follows. If $0<b-a$ then $A=0$ and $B=b+a$, so that the result already holds for the largest zeros. For the smallest zeros we use the interlacing property to observe that $x_{1, n}(\alpha)<x_{1,1}(\alpha)$. Hence, since $\varphi$ is increasing, we obtain

$$
\liminf _{n \rightarrow \infty} \frac{x_{1, n}(\alpha)}{\varphi(n)} \leq 0
$$

which gives the desired result for the smallest zeros. Similarly, when $0>b+a$, then $A=b-a$ and $B=0$ so that the result holds for the smallest zeros, whereas for the largest zeros the interlacing property gives $x_{n, n}(\alpha) \geq x_{1,1}(\alpha)$, from which

$$
\limsup _{n \rightarrow \infty} \frac{x_{n, n}(\alpha)}{\varphi(n)} \geq 0,
$$

giving the desired result again. 
The analog of the $(C,-1)$-summability result of Theorem 2 is given by Theorem 6. Suppose $\lim _{n \rightarrow \infty} a_{n}(\alpha) / \varphi(n)=a / 2 \geq 0$ and $\lim _{n \rightarrow \infty} b_{n}(\alpha) / \varphi(n)=$ $b \in \mathbb{R}$, where $\varphi$ is a log-slowly varying function. Let $f$ be absolutely continuous in an interval $\Delta$. If, for every $n>0,\left[x_{1, n}(\alpha) / \varphi(n), x_{n, n}(\alpha) / \varphi(n)\right] \subseteq \Delta$, then

$$
\begin{array}{r}
\lim _{n \rightarrow \infty} \sum_{j=1}^{n} f\left(x_{j, n}(\alpha) / \varphi(n)\right)-\sum_{j=1}^{n-1} f\left(x_{j, n-1}(\alpha) / \varphi(n)\right) \\
= \begin{cases}\frac{1}{\pi} \int_{b-a}^{b+a} \frac{f(t) d t}{\sqrt{a^{2}-(t-b)^{2}}}, & \text { if } a>0, \\
f(b), & \text { if } a=0 .\end{cases}
\end{array}
$$

For continuously differentiable functions $f$ and $a>0$, Theorem 6 is given in [10, Theorem 2.4, p. 14]. The extension to absolutely continuous functions and $a \geq 0$ can be made just as in the proof of Theorem 2 by using Lemma 5 . Note that by (14), $(A, B) \subseteq \Delta$.

The analog of Theorem 4 for log-slowly varying recurrence coefficients is in terms of

$$
\Omega_{n}^{\varphi}(\alpha, g) \stackrel{\text { def }}{=} \frac{1}{\varphi(n)} \int_{x_{1, n}(\alpha)}^{x_{n, n}(\alpha)}\left[2 F_{n}(\alpha, x)-2 F_{n-1}(\alpha, x)-1\right] g\left(\frac{x}{\varphi(n)}\right) d x
$$

which is a natural analog of (10).

Theorem 7. Suppose $\lim _{n \rightarrow \infty} a_{n}(\alpha) / \varphi(n)=a / 2 \geq 0$ and $\lim _{n \rightarrow \infty} b_{n}(\alpha) / \varphi(n)=$ $b \in \mathbb{R}$, where $\varphi$ is a log-slowly varying function. Let $g$ be integrable in an interval $\Delta$. If, for every $n>0,\left[x_{1, n}(\alpha) / \varphi(n), x_{n, n}(\alpha) / \varphi(n)\right] \subseteq \Delta$, then

$$
\lim _{n \rightarrow \infty} \Omega_{n}^{\varphi}(\alpha, g)=\left\{\begin{array}{cc}
\frac{2}{\pi} \int_{b-a}^{b+a} g(t) \arcsin \left(\frac{t-b}{a}\right) d t-\int_{A}^{b-a} g(t) d t+\int_{b+a}^{B} g(t) d t, \\
\text { if } a>0, \\
-\int_{A}^{b} g(t) d t+\int_{b}^{B} g(t) d t, & \text { if } a=0,
\end{array}\right.
$$

where $[A, B]$ is the convex hull of $[b-a, b+a] \cup\{0\}$.

The proof of Theorem 7 goes along the lines of the proof of Theorem 4 with minor modifications.

\section{ACKNOWLEDGMENT}

We thank Dennis Stanton for bringing S. Kerov's manuscript [2] to our attention.

\section{REFERENCES}

1. E. W. Cheney, Introduction to approximation theory, Second Edition, Chelsea, New York, 1982.

2. S. Kerov, Separation of roots of orthogonal polynomials and the limiting shape of generic large Young diagrams, Mathematics, No. 8, Department of Mathematics, The University of Trondheim, Trondheim, Norway, 1992, pp. 1-23.

3. _ The asymptotics of interlacing sequences and the growth of continual Young diagrams, preprint, 1992. 
4. $\ldots$, The asymptotics for interlacing roots of orthogonal polynomials, Algebra i Analiz (1993) (to appear). (Russian)

5. Transition probabilities for continual Young diagrams and the Markov moment problem, Funktsional. Anal. i Prilozhen. 27 (1993), 32-49; English transl., Functional Anal. Appl. 27 (1993), 104-117.

6. P. Nevai, Distribution of zeros of orthogonal polynomials, Trans. Amer. Math. Soc. 249 (1979), 341-361.

7. __ Orthogonal polynomials, Mem. Amer. Math. Soc., vol. 213, Providence, RI, 1979.

8. G. Szegö, Orthogonal polynomials, Fourth Edition, Colloq. Publ., vol. 23, Amer. Math. Soc., Providence, RI, 1975.

9. W. Van Assche, Asymptotics for orthogonal polynomials, Lecture Notes in Math., vol. 1265, Springer-Verlag, Berlin, 1987.

10. - Orthogonal polynomials on non-compact sets, Acad. Analecta, Meded. Konink. Acad. Wetensch. Lett. Sch. Kunsten België 51 (1989), Nr. 2, 1-36.

11. __ Asymptotics for orthogonal polynomials and three-term recurrences, Orthogonal Polynomials: Theory and Practice (P. Nevai, ed.), NATO-ASI Series C 294, Kluwer, Dordrecht, 1990, pp. 435-462.

Department of Mathematics, The Ohio State University, 231 West 18th Avenue, Columbus, Оhiо 43210-1174

E-mail address: nevai@math.ohio-state.edu and nevaiCohstpy.bitnet

Department of Mathematics, Katholieke Universiteit leuven, Celestijnenlaan 200 B, B-3001 Heverlee (Leuven), Belgium

E-mail address: fgaee03ecc1.KULeuven.ac.be 